\title{
Participación del ingreso laboral en el ingreso total en América Latina, 1990-2010
}

\author{
Martín Abeles, Verónica Amarante y Daniel Vega
}

En este artículo se analiza la participación de los ingresos laborales en el ingreso total

en los países de América Latina durante las últimas dos décadas. Primero se considera la participación de la masa de salarios en el producto interno bruto (PIB), para luego incorporar los ingresos laborales de los trabajadores independientes. Los resultados indican que tanto el peso de la masa salarial como el del total de ingresos laborales en el PIB han caído durante el período en la mayoría de los países de la región, aunque con algunas excepciones. La merma en la desigualdad del ingreso laboral en la última década no ha estado acompañada, en términos generales, de una mayor participación de los ingresos laborales en el PIB. Esto implica que la mejora en la distribución personal de los ingresos no se ha producido en conjunto con una mejora en la distribución funcional.

PALABRAS CLAVE

CLASIFICACIÓN JEL

AUTORES
Empleo, ingresos, producto interno bruto, distribución del ingreso, medición, datos estadísticos, América Latina

D33, E25, J31

Martín Abeles es director de la Oficina de la cepal en Buenos Aires. martin.abeles@cepal.org

Verónica Amarante es directora de la Oficina de la CEPAL en Montevideo.veronica.amarante@cepal.org

Daniel Vega es asistente estadístico en la Oficina de la cepal en Buenos Aires. daniel.vega@cepal.org 


\section{I}

\section{Introducción}

En las últimas décadas, la amplia mayoría de los estudios sobre desigualdad se concentraron en el análisis de la distribución del ingreso entre las personas y los hogares. Si bien en los últimos años el estudio de la distribución funcional del ingreso tendió a cobrar mayor relevancia en los países avanzados — en buena medida a raíz de la caída de la participación de los salarios en el ingreso experimentada en esos países durante los últimos 30 años-, el tema parece no revestir el mismo interés en los países de la región latinoamericana. En este artículo se plantea la necesidad de recuperar el estudio sistemático de la distribución funcional del ingreso, y se discuten algunas de las limitaciones vinculadas con la disponibilidad de información, que habitualmente se encuentran para hacerlo, aspecto muy relevante en los países de América Latina.

Se analiza la evolución reciente de la participación de los ingresos laborales en el pIB total de las economías de la región. La evidencia empírica que se presenta se estructura en torno de dos ejes. Por una parte, se considera la información existente sobre la participación de la masa salarial en los países de la región durante el período comprendido entre 1990 y 2011. Por otra, se corrige la participación de la masa salarial en el PIB, incorporando el ingreso del trabajo independiente. El ajuste se realiza sobre la base de dos metodologías. En primera instancia se realiza un ajuste - muy difundido en la literatura- que consiste en asumir que todos los trabajadores independientes reciben un ingreso laboral similar al salario promedio de la economía. En segundo lugar se realiza un ajuste más riguroso, utilizando datos de las encuestas de hogares. El primer paso de este segundo ajuste consiste en estimar, dentro del ingreso mixto de los trabajadores independientes (tanto trabajadores por

$\square$ Los autores agradecen a Maximiliano García y Martin Schmitt por su excelente labor como asistentes de investigación para este trabajo. cuenta propia como patrones), la parte correspondiente al ingreso laboral, empleando información de las encuestas continuas de hogares. Una vez que se identifica la masa de ingresos laborales correspondientes al trabajo independiente, se calcula la relación de proporcionalidad que guarda con la masa salarial, recurriendo también a la información de las encuestas de hogares. A partir de esta relación, se ajusta la masa salarial relevada por los sistemas de cuentas nacionales para obtener una estimación del peso del total de ingresos laborales en el PIB. Sobre la base de estas dos aproximaciones metodológicas, en el artículo se presentan estimaciones novedosas acerca de la importancia de los ingresos laborales en América Latina, ilustrando además con respecto a su evolución y reflexionando sobre los problemas y limitaciones que existen para el estudio de la distribución funcional del ingreso en la región.

El artículo se organiza de la siguiente manera: en primer lugar se discuten el enfoque funcional y personal para el estudio de la distribución del ingreso (sección II). A continuación se describen aspectos vinculados con la medición de la participación del ingreso laboral en el ingreso total (sección III), para luego sintetizar los estudios recientes sobre distribución funcional del ingreso (sección IV). En la sección siguiente se aborda la discusión de aspectos metodológicos, presentando las fuentes de información disponibles para este tipo de estudios en América Latina y describiendo las opciones metodológicas adoptadas en este trabajo (sección V). Se analiza a continuación la evolución de la participación de la masa salarial en el ingreso total en América Latina (sección VI). Luego de subrayar la importancia del trabajo independiente en América Latina (sección VII), se presentan los resultados que surgen al corregir esta masa salarial de manera de incorporar el ingreso por trabajo correspondiente a los trabajadores independientes (sección VIII). Finalmente, se presentan los comentarios finales en la sección IX. 


\section{II}

\section{Distribución funcional y distribución personal del ingreso}

Los economistas clásicos han hecho hincapié en el análisis de la relación entre la distribución funcional del ingreso y el proceso productivo y de formación de capital. En su ya célebre comentario al comienzo de sus Principios de economía política y tributación, David Ricardo sostenía que la determinación de las leyes que rigen la división del producto entre trabajadores, capitalistas y terratenientes constituye el principal problema de la economía política (Ricardo, 1973). Como sostienen Serrano y Medeiros (2001), la noción de excedente económico tal y como era concebida en el enfoque clásico permeó en diferente medida en varios de los trabajos seminales en el campo del desarrollo económico, como en el caso del conocido modelo de economía dual de Arthur Lewis (1954). El análisis de la relación entre el desarrollo económico y la caída del empleo en actividades tradicionales o de subsistencia (típicamente en áreas rurales, véase Bhaduri, 1983) o la propia relación entre la generación del excedente y la acumulación de capital desde una perspectiva sectorial y estructural (Rodríguez, 2006) implica, de una u otra manera, el análisis de la distribución del excedente entre los distintos sectores o clases sociales.

El énfasis clásico en la distribución funcional del ingreso sobrevivió a la revolución marginalista de fines del siglo XIX, aunque dentro de un marco conceptual y metodológico diferente, en función del cual cada factor de la producción (las clases sociales de los autores clásicos) se apropiaba de una parte del producto sobre la base de su contribución marginal al proceso productivo. En esa línea, un indicador fundamental es el peso de la masa salarial en el producto total generado por la economía. Es con la consolidación de la economía neoclásica hacia mediados de la década de 1950, y su acento en el análisis fundado en el estudio del comportamiento individual de los agentes económicos, que se aprecia un viraje en el énfasis de los economistas de la corriente principal desde la distribución funcional a la distribución personal del ingreso (Goldfarb y Leonard, 2005). En sus orígenes, el interés por el estudio de la distribución personal del ingreso en detrimento de la distribución funcional enfrentó resistencias de los economistas poskeynesianos y neoricardianos, quienes reivindicaban la preeminencia de la discusión distributiva desde una perspectiva funcional, no solo con fundamentos analíticos ${ }^{1}$, sino con el objetivo explícito de hacer hincapié en la centralidad del conflicto social entre capitalistas y trabajadores en el desenvolvimiento de las economías capitalistas ${ }^{2}$. Buscaban así subrayar la idea de que las elecciones individuales se encuentran fuertemente condicionadas por el lugar que ocupa cada persona en la estratificación social. A partir de la década de 1960, el viraje hacia la distribución personal resulta ostensible y el análisis de la distribución funcional del ingreso queda relegado a un segundo plano (Atkinson, 2009).

Diversos factores históricos o institucionales han motivado el creciente interés por la distribución personal del ingreso. La complejidad de los procesos productivos modernos, así como la importante heterogeneidad dentro de los grupos vinculados a los distintos factores de producción, contribuyen a explicar por qué los análisis sobre desigualdad han tendido a centrarse en la distribución personal. Los individuos y hogares pueden obtener ingresos ligados a distintos factores productivos, y además dentro de un mismo grupo (por ejemplo, los asalariados) la desigualdad puede ser muy alta. Desde una perspectiva institucional, con la consolidación de los estados de bienestar surge la necesidad de identificar con mayor precisión a los grupos sociales más vulnerables, principales destinatarios de la asistencia pública, y de examinar con mayor rigurosidad la distribución del ingreso en el interior de la clase trabajadora. El enfoque personal permitía profundizar el análisis del impacto de la acción redistributiva del Estado, al considerar el efecto de impuestos y transferencias en los ingresos sobre la base de información estadística de las encuestas de hogares. En tal sentido, la proliferación del enfoque personal estuvo también relacionada con la recolección de información a nivel de hogares por medio de encuestas específicas, y con los avances metodológicos y tecnológicos en materia de investigación aplicada.

\footnotetext{
1 Remarcaban, entre otras cosas, la necesidad de diferenciar las propensiones al ahorro entre las distintas clases sociales en el análisis macroeconómico.

2 Autores como Joan Robinson, Nicholas Kaldor y Luigi Pasinetti desarrollaron argumentaciones en este sentido.
} 
Podría decirse, también, que el análisis de la evolución de la distribución funcional del ingreso tendió a ser relegado a consecuencia de la propia estabilidad en los datos sobre participación asalariada en el ingreso durante la segunda posguerra — una constatación empírica que llegó a identificarse como un hecho estilizado de las economías capitalistas (Kaldor, 1961)—. Esta regularidad empírica se vería a su vez reforzada por la consolidación de la teoría neoclásica del crecimiento y la idea de que las posibilidades de producción de una economía pueden ser reflejadas mediante una función de producción Cobb-Douglas ${ }^{3}$, que implica una elasticidad de sustitución unitaria entre trabajo y capital, y participaciones factoriales constantes.

Sin embargo, el tema recobró relevancia en los últimos años. Desde el campo académico, diversos autores de extracción poskeynesiana, estructuralista o de ambas, sistematizaron el estudio del impacto de cambios en la distribución funcional del ingreso en la demanda agregada y el crecimiento económico ${ }^{4}$. Desde un punto de vista empírico, tendió a perder validez la supuesta constancia de la distribución funcional del ingreso, al observarse una trayectoria declinante de la participación asalariada en el ingreso en prácticamente todos los países industrializados desde comienzos de la década de 1980 (véase la sección III más adelante). La novedad concitó incluso la atención académica, como se advierte en varios estudios recientes ${ }^{5}$. En los países en desarrollo, el tema también suscitó mayor interés. En América Latina y el Caribe, por ejemplo, con el auge de los precios de los productos básicos de exportación, vuelve a cobrar centralidad la magnitud y el origen sectorial del excedente económico para la trayectoria del proceso de desarrollo (CEPAL, 2012a) — sobre todo en los países de América del Sur-, y la estimación de la renta asociada a la extracción de los recursos naturales se convierte en un insumo relevante para la toma de decisiones de política (Campodónico, 2008; CEPAL, 2013).

\footnotetext{
${ }^{4}$ Véanse, por ejemplo, Bhaduri y Marglin (1990) y Taylor (1991) con respecto a los aportes seminales. Para una reseña de esa literatura, véase Abeles y Toledo (2011).

5 Véanse, por ejemplo, Bentolila y Saint-Paul (2003); Gollin (2002); Serres, Scarpetta y Maisonneuve (2001); Feldstein (2008); FMI (2007); Comisión Europea (2007); oIT (2008); Ellis y Smith (2010).
}

\section{III}

\section{La medición de la participación de los ingresos laborales en el ingreso total}

La naturaleza del proceso de generación de ingresos a partir de la actividad económica se refleja en la cuenta de generación del ingreso del Sistema de Cuentas Nacionales (SCN). En esta cuenta se aprecia cómo se distribuye el valor agregado bruto entre los trabajadores y los propietarios del capital (incluida la tierra y otros recursos naturales cuya renta pueda ser apropiada por privados), y el gobierno. De acuerdo con la revisión de 1993, se considera al valor agregado como recurso en el proceso de generación del ingreso, y como uso a las remuneraciones a los asalariados y los impuestos a los productos y a la producción, netos de subsidios. El saldo contable es el excedente de explotación o ingreso mixto, según sea la naturaleza de la cuenta. El ingreso mixto refleja el excedente derivado de las actividades productivas de las empresas no constituidas en sociedades, es decir, de los hogares ${ }^{6}$. Ese ingreso mixto contiene entonces implícitamente un elemento de remuneración al trabajo y un elemento de remuneración al capital involucrado en la actividad productiva que se analiza. En el mejor de los casos, las cuentas nacionales brindan información agregada sobre estos dos elementos componentes del ingreso mixto, y no pueden identificarse por separado

\footnotetext{
${ }^{6}$ Se trata, fundamentalmente, de trabajadores independientes o por cuenta propia (por ejemplo, un vendedor ambulante).
} 
el rendimiento del trabajo y el del capital ${ }^{7}$. Aquí radica una de las principales limitaciones para la medición de la participación de los salarios en el ingreso, limitación que es sobre todo relevante en los países en desarrollo, donde el trabajo independiente resulta preponderante. En efecto, el total del ingreso de los trabajadores independientes suele quedar incluido en ese ingreso mixto, sin distinguir remuneraciones al trabajo y al capital.

La mayor parte de las veces, cuando se analiza la participación laboral en el ingreso total, se considera simplemente el cociente entre las remuneraciones a los asalariados y el producto interno bruto (PIB) total ${ }^{8}$.

\footnotetext{
${ }^{7}$ En algunos países de la región, como el Perú entre 1950 y 1965, se publicaba el ingreso de los trabajadores independientes como parte de sus estadísticas de cuentas nacionales, pero esta información fue discontinuada en el marco de la adecuación a las propuestas de las Naciones Unidas para la normalización de los Sistema de Cuentas Nacionales.Véase [en línea] http://institutodelperu.org.pe/descargas/ Publicaciones/De\%20otras\%20entidades/DOC/1966_webb_cuentas_ nacionales_del_peru.pdf.

8 También los estudios de participación laboral basados en datos provenientes de encuestas a establecimientos o de censos industriales tienen una limitación de este tipo, ya que se mide la actividad de las firmas por sobre un cierto umbral (ya sea de producción o de número de ocupados), y por lo tanto, se excluyen las empresas pequeñas o familiares (una descripción detallada de las bases de datos que compilan este tipo de información puede encontrarse en Rodríguez y Ortega, 2006).
}

Este cuociente puede expresarse a precio de mercado, o a costo de factores, dependiendo de si se incluyen o no los impuestos sobre producción y productos netos de subvenciones en la medición del PIB. La simple consideración de esta razón muestra que la participación salarial varía de manera muy importante entre los países, desde un 14\% en Nigeria hasta el 59\% en Suiza, y los países de la región tienden a presentar menores valores de este indicador (véase el gráfico 1).

Una regularidad que surge al analizar el peso de los salarios en el PIB a nivel internacional es la asociación positiva que muestran con el nivel de riqueza de los países. La participación de los ingresos salariales en el PIB se incrementa en los países más ricos (véase el gráfico 2). Sin embargo, esta relación puede ser engañosa, ya que el hecho de que la participación del trabajo aumente con el PIB per cápita puede vincularse con el mayor peso de la economía informal en los países menos desarrollados. Tanto las comparaciones temporales como entre países pueden, por lo tanto, tener importantes sesgos. A nivel temporal, las comparaciones estarían afectadas si, como sugiere la evidencia, los ingresos de los asalariados y los ingresos del trabajo independiente reaccionaran de manera diferente al ciclo económico. Con respecto a las comparaciones entre países, estas

GRÁFICO 1

Participación de los salarios en el PIB, a precios de mercado alrededor de $\mathbf{2 0 1 0}$ (En porcentajes)

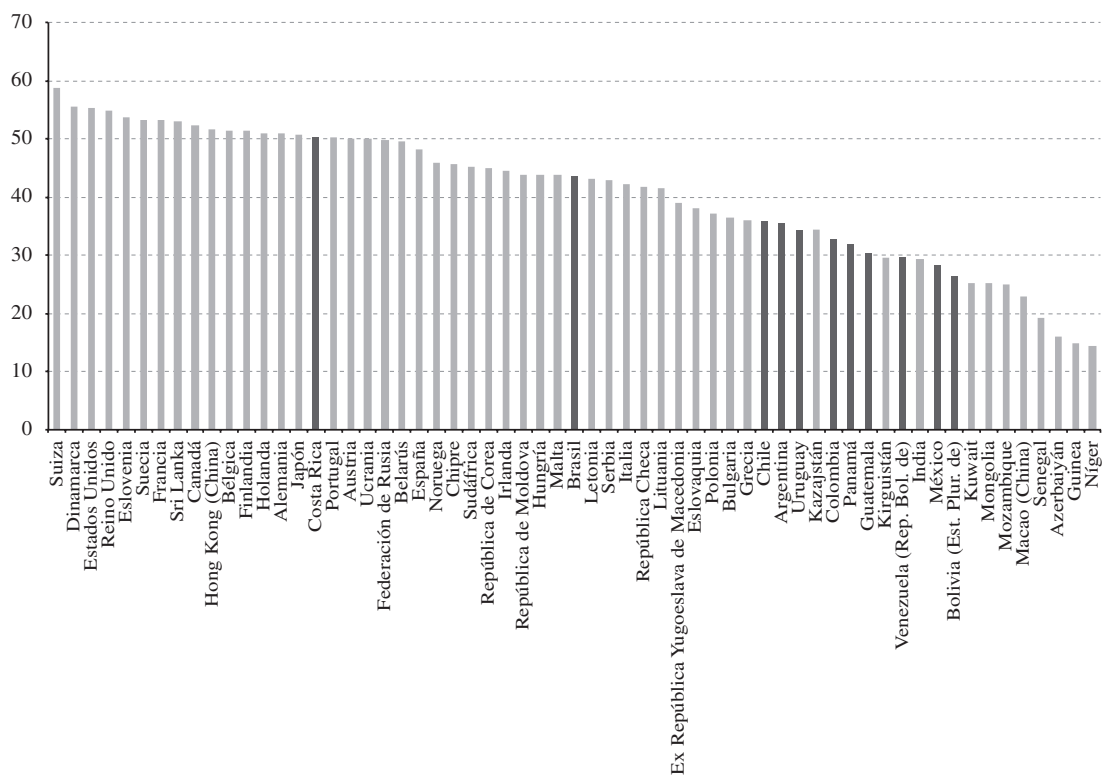

Fuente: elaboración propia sobre la base de datos de la División de Estadística de las Naciones Unidas (UNSD).

PIB: producto interno bruto. 
estarían marcadamente sesgadas por la no inclusión de los ingresos del trabajo independiente. Ello llevaría a una subestimación de la participación del trabajo en el ingreso total, que será mayor cuanto mayor sea el peso del trabajo independiente y, por lo tanto, de la masa de ingresos de esos trabajadores no incluidos.

GRÁFICO 2

Participación de los salarios en el PIB y PIB per cápita, a precios de mercado, 2010 (En porcentajes)

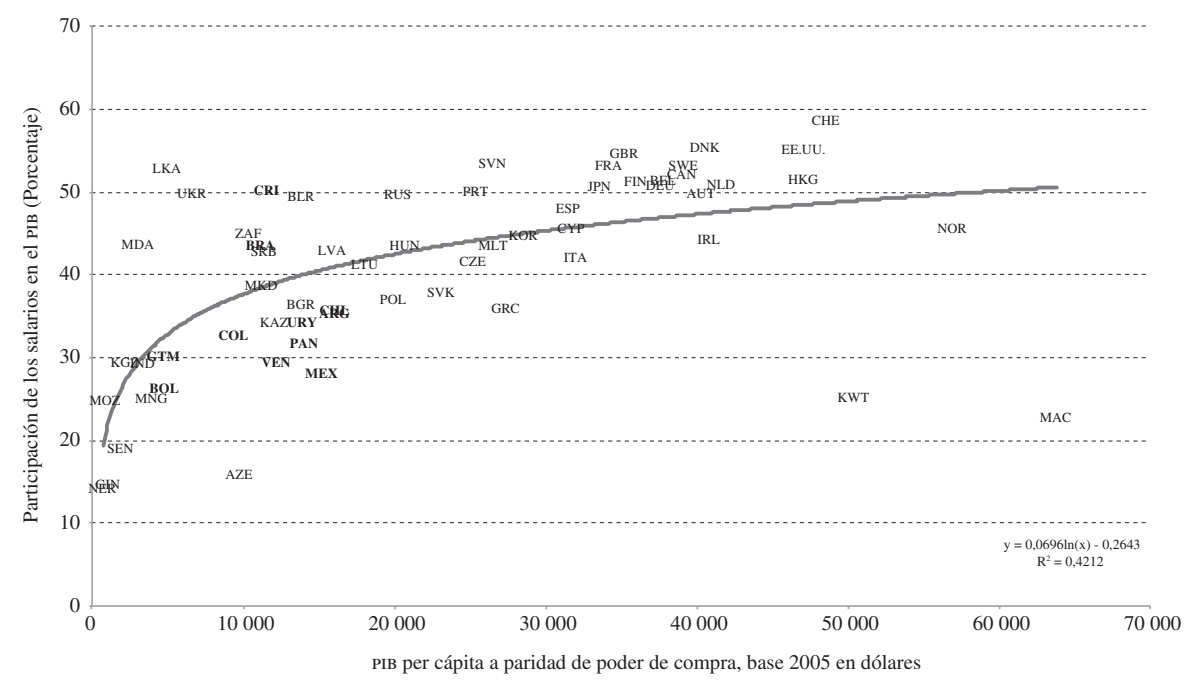

Fuente: elaboración propia sobre la base de datos de la División de Estadística de las Naciones Unidas (UNSD) y Fondo Monetario Internacional (FMI), World Economic Outlook Database.

PIB: producto interno bruto.

CHE: Suiza, DNK: Dinamarca, EE.UU.: Estados Unidos, GBR: Reino Unido, SVN: Eslovenia, FRA: Francia, SWE: Suecia, CAN: Canadá, FIN: Finlandia, BEL: Bélgica, LKA: Sri Lanka, JPN: Japón, DEU: Alemania, HKG: Hong Kong (China), NLD: Holanda, UKR: Ucrania, CRI: Costa Rica, BLR: Bielorrusia, RUS: Federación de Rusia, PRT: Portugal, ESP: España, AUT: Austria, MDA: República de Moldova, ZAF: Sudáfrica, BRA: Brasil, SRB: Serbia y Montenegro, LVA: Letonia, HUN: Hungría, KOR: República de Corea, CYP: Chipre, IRL: Irlanda, CZE: República Checa, ITA: Italia, LTU: Lituania, NOR: Noruega, MLT: Malta, MKD: Macedonia, SVK: Eslovaquia, POL: Polonia, BGR: Bulgaria, GRC: Grecia, CHL: Chile, ARG: Argentina, URY: Uruguay, PAN: Panamá, COL: Colombia, VEN: República Bolivariana de Venezuela, MEX: México, GTM: Guatemala, BOL: Estado Plurinacional de Bolivia, KWT: Kuwait, MOZ: Mozambique, MAC: Macao (China), MNG: Mongolia, SEN: Senegal, AZE: Azerbaiyán, GIN: Guinea, NER: Nigeria.

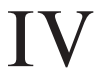

\section{Estudios recientes sobre participación de los ingresos laborales en el ingreso total y sus determinantes}

Los estudios recientes sobre distribución funcional del ingreso coinciden en señalar un cambio significativo en las últimas tres décadas. En contraste con lo observado en los llamados "treinta gloriosos años" de la posguerra, en los países de la Organización para la Cooperación y el Desarrollo Económicos (OCDE) se ha producido una caída de la participación de la masa de ingresos laborales en el total del ingreso. La tendencia es menos homogénea en los países en desarrollo y en las economías emergentes, aunque en la mayoría se advierte también una caída en la participación del ingreso laboral. Ejemplos recientes que brindan esta evidencia son Stockhammer (2013) y OIT (2011 y 2013). En estos estudios se considera la evolución de la masa salarial ajustada, que surge al multiplicar la 
compensación promedio de los asalariados por el número de trabajadores de la economía. De esta forma, se está incorporando a los trabajadores independientes a través del supuesto de que su remuneración es similar a la de los trabajadores dependientes.

La caída en la participación del ingreso laboral en el ingreso total no se deriva, en términos generales, de cambios en la estructura de la actividad económica, que implicarían pérdida de importancia de los sectores con alta participación salarial en detrimento de los sectores con menor participación (efectos de desplazamiento). Más bien, obedece a la merma en la relevancia de la masa de salarios que se produce en el interior de ciertos sectores (OIT, 2011). En particular, se observan importantes caídas de esta razón en intermediación financiera y manufacturas de alta y mediana tecnología, y un retroceso menos pronunciado en servicios, construcción y manufactura de bajo contenido tecnológico. También se ha encontrado que la tendencia descendente en la participación de los ingresos laborales ajustados en los países desarrollados se debe, sobre todo, al deterioro de la participación de los ingresos de los trabajadores de calificación baja y media, mientras que respecto de los trabajadores de alta calificación, la participación de los ingresos laborales tiende a aumentar (oIT, 2013).

En los últimos años ha surgido una literatura bastante abundante, en que se intenta relacionar la evolución reciente de la participación del ingreso laboral con las reformas estructurales implementadas en las décadas recientes. Se analiza el posible vínculo con diversos fenómenos tales como: la deslocalización global de los procesos productivos, la desregulación de los mercados laborales, el predominio cada vez mayor de los mercados financieros y su desregulación, los cambios en las instituciones y el grado de sindicalización, entre otros
(Bentolila y Saint-Paul, 2003; Bernanke y Gürkaynak, 2002; Fichtenbaum, 2009; Gollin, 2002; Harrison, 2002; Hogrefe y Kappler, 2012; FMI, 2007; Jayadev, 2007; Rodríguez y Ortega, 2006). No se advierte, sin embargo, un abordaje sistemático del vínculo entre la evolución de la distribución funcional y personal del ingreso. Mientras que los estudios de distribución funcional se abordan mediante un enfoque macroeconómico, aquellos que se centran en la distribución personal la conciben como un fenómeno microeconómico, explicado básicamente por la distribución de las características personales de los individuos. Un intento de relacionar ambos enfoques se encuentra en el trabajo de Daudey y García-Peñalosa (2007), que aporta evidencia econométrica de que la baja participación de la masa salarial en el producto tiene un efecto negativo y significativo en la desigualdad personal del ingreso.

Para comprender mejor los determinantes de la desigualdad del ingreso y la vinculación entre desigualdad personal y funcional, es necesario primero tener un diagnóstico acertado de la distribución funcional del ingreso y su evolución. Existen análisis desde una perspectiva factorial para algunos países de la región, entre ellos Lindenboim (2008), Lindenboim, Kennedy y Graña (2010) y Graña (2007) para la Argentina; Hernández Laos (1998) para México; PNUd (2010) y Amarante y Vigorito (2011) para el Uruguay; y Ministerio de Planificación y Cooperación (2000) para Chile. Sin embargo, no hay análisis en que se combine una perspectiva comparativa en la región, y a su vez se incorpore de manera sistemática y comparable el ingreso del trabajo independiente. En el presente artículo se procura avanzar en esa línea, cuyo abordaje puede parecer sencillo, si bien enfrenta distintos problemas vinculados a la información disponible, como se detalla a continuación.

\section{V}

\section{Aspectos metodológicos}

\section{La información disponible en América Latina}

En la región, la elaboración de las estadísticas de cuentas nacionales está a cargo de los bancos centrales o de los institutos nacionales de estadística ${ }^{9}$. No todos los países

\footnotetext{
9 Los institutos de estadística son los encargados de recopilar la información en la Argentina, Bolivia (Estado Plurinacional de), el Brasil, Colombia, México, Panamá y el Perú. Los bancos centrales
}

presentan públicamente la información del ingreso mixto de manera desagregada. Dicha información se encuentra disponible en la región para la Argentina, el Brasil, Chile, Colombia, Guatemala, Honduras, México, Nicaragua, Panamá, el Paraguay, el Perú, el Uruguay y Venezuela

lo hacen en Chile, Costa Rica, el Ecuador, El Salvador, Guatemala, Honduras, Nicaragua, el Paraguay, República Dominicana, el Uruguay y Venezuela (República Bolivariana de). 
(República Bolivariana de), aunque en la mayoría de los casos solo para el período más reciente (principalmente a partir del último cambio de base) y en algunos casos sin la desagregación a nivel de rama de actividad ${ }^{10}$. Esta información es compilada por los bancos de datos de la Comisión Económica para América Latina y el Caribe (CEPAL) y de la División de Estadísticas de las Naciones Unidas (UNSD por sus siglas en inglés) ${ }^{11}$.

Los datos de la CEPAL se encuentran disponibles en CEPALSTAT, base de datos que incluye información correspondiente a los últimos años para 14 de los 18 países de la región (sin contar a Cuba ni a Haití), y sin discriminar el ingreso mixto, que es presentado de manera consolidada con el excedente de explotación (incluso en los casos en que los países presentan dicha información de manera desagregada). El detalle de la información disponible en este banco de datos se presenta en el cuadro A.1 del anexo. Dicha base de datos tiene la ventaja de contener información de largo plazo, aunque en la práctica las series temporales presentan importantes interrupciones debido a los cambios de base u otras alteraciones metodológicas.

La UNSD incluye información para una gran cantidad de países, entre ellos 16 países de la región, distinguiendo el ingreso mixto del excedente de explotación e incluyendo una apertura de las series por sector de actividad ${ }^{12}$. Sin embargo, los datos abarcan un período más acotado de tiempo (solo para 7 de los 16 países contiene datos desde la década de 1970). El detalle de la información sobre América Latina incluida en la UNSD se presenta en el cuadro A.2 del anexo.

En este trabajo se recoge la información contenida en la base de datos CEPALSTAT, y se complementa con la información que proviene de los organismos oficiales correspondientes en el caso de los países no incorporados a CePalstat (Argentina, Costa Rica y Guatemala). En el caso del Uruguay, dado que la información del SCN llega hasta el año 2005, la razón de participación salarial fue actualizada utilizando la evolución del índice medio de salario nominal, la tasa de empleo y el PIB a costo de factores.

\footnotetext{
10 Algunos países cuentan con información de los ingresos mixtos antes de 1990 en sus sistemas de cuentas nacionales.

11 Existen otras fuentes de información para analizar el peso de los salarios en el PIB, pero no basadas en datos de cuentas nacionales, sino en encuestas a establecimientos o censos. Las dos principales bases de datos de este tipo son la de la Organización de las Naciones Unidas para el Desarrollo Industrial (UNIDO) y la de la Organización de Cooperación y Desarrollo Económicos (OCDE). Detalles sobre estas bases de datos pueden encontrarse en Rodríguez y Ortega (2006).

${ }^{12}$ En este total no se incluye al Ecuador, cuyos datos llegan solamente hasta 1991. En la apertura por sector de actividad, la información de ingreso mixto no siempre se encuentra disponible.
}

\section{Metodologías para la estimación del ingreso laboral total}

Para evitar los sesgos en las mediciones de participación laboral discutidos en la sección III — que derivaron de no incluir el total de los ingresos laborales en el análisis, sino solo los salariales-, es necesario estimar el ingreso laboral correspondiente a los trabajadores independientes.

Un primer problema que es preciso afrontar entonces es cómo separar, dentro del ingreso mixto, la parte correspondiente a remuneraciones al trabajo y la que refleja retornos al capital. El primer componente debería agregarse a la remuneración a los asalariados para obtener la verdadera participación laboral en el ingreso generado en la economía. Distintas aproximaciones han sido sugeridas para realizar esta corrección. Una posibilidad es efectuar estimaciones suponiendo que los trabajadores independientes reciben una remuneración similar al salario promedio. Gollin (2002) realiza ajustes de este tipo para un amplio conjunto de países, y concluye que una parte significativa de las diferencias en la participación de los ingresos del trabajo en el ingreso total entre países ricos y pobres obedece a errores metodológicos ocasionados por no incluir los ingresos del trabajo independiente. Los estudios de Stockhammer (2013) y OIT (2011 y 2013) se basan también en una corrección de este tipo. En el presente trabajo se realizan estimaciones sobre la base de dos metodologías, la primera consiste en considerar que los trabajadores independientes reciben una remuneración promedio similar a la de los asalariados.

Una solución más rigurosa consiste en intentar separar, dentro del ingreso mixto y respecto de los países que lo computan separadamente, la parte correspondiente a la remuneración del trabajo y los retornos al capital. Para ello, se puede simular el ingreso laboral que los trabajadores independientes recibirían según sus características personales y sector de actividad de la economía (en lugar de tomar el salario promedio para todos). Una solución de este tipo es adoptada por Young (1995) para los países de Asia a partir de información censal, asumiendo que los trabajadores independientes ganan lo mismo que los asalariados con similar edad, sexo, nivel educativo y sector de actividad. Aun cuando no se disponga de información oficial sobre el ingreso mixto, se puede corregir la estimación del peso de los ingresos laborales. Para ello, primero se calcula en la encuesta de hogares la relación entre la masa salarial y el ingreso del trabajo independiente, y luego se aplica ese coeficiente al ingreso salarial de las cuentas nacionales.

En la actualidad, estas estimaciones pueden realizarse utilizando información de las encuestas continuas de 
hogares. Esta es la segunda opción metodológica que se adopta en este artículo. Se estima la relación entre la masa de ingresos salariales y la masa de ingresos por trabajo de los trabajadores independientes sobre la base de los microdatos de las encuestas continuas de hogares, y se emplea esa relación para corregir la participación de la masa salarial en el PIB.

Como primer paso, se calcula el ingreso laboral que los trabajadores independientes recibirían teniendo en cuenta sus características personales y el sector de actividad de la economía, y suponiendo que recibirían remuneraciones similares a las de los asalariados. Para ello, se parte de la estimación de ecuaciones salariales para los asalariados privados, incluidas como variables dependientes el sexo, la edad y su expresión cuadrática, los años de escolaridad, y variables binarias que distinguen por rama de actividad. Los coeficientes obtenidos en estas ecuaciones salariales son aplicados a las características de los trabajadores independientes, a objeto de disponer de predicciones de los ingresos laborales para cada trabajador independiente incluido en la encuesta. Cuando estas predicciones de ingreso laboral de los trabajadores independientes son inferiores al ingreso que reportan en las encuestas de hogares, se asume que esa diferencia corresponde a la retribución al capital. Por lo tanto, se toma la predicción como ingreso laboral de esos trabajadores independientes. En caso de que la predicción del ingreso sobrepase al ingreso declarado en las encuestas de hogares, se toma todo el ingreso declarado en dichas encuestas como ingreso laboral de los trabajadores independientes. A partir de este nuevo vector de ingresos laborales de los trabajadores independientes, es posible establecer una relación de proporcionalidad entre la masa de ingresos laborales de los trabajadores independientes y la masa salarial (también reportada en las encuestas de hogares). Esa relación es aplicada a los datos de la masa salarial del SCN con el propósito de lograr una estimación final de la masa de ingresos laborales, que se compara con el PIB.

\section{VI \\ Participación de la masa salarial en el ingreso total en América Latina}

La información disponible a partir de los datos de cuentas nacionales de los países permite un primer análisis de la evolución de la participación salarial en el ingreso nacional de los países de la región. Se trata, como ya se discutió, de una aproximación inexacta, ya que solo incluye el ingreso de los trabajadores asalariados. Por otra parte, las series temporales para la región presentan importantes saltos en los momentos de cambio del año base de las cuentas nacionales, ante lo cual se optó por considerar las series temporales continuas disponibles para los países en el período comprendido entre 1990 y el último año disponible (variable según el país). Se considera la razón entre remuneración del trabajo asalariado y PIB a costo de factores ${ }^{13}$. Se toma la información contenida en CEPALSTAT y se complementa con la obtenida de los organismos oficiales correspondientes en el caso de los países no incorporados a CEPALSTAT (Argentina, Costa Rica y Guatemala). En el caso del Uruguay, dado

\footnotetext{
13 Las mediciones a costo de factores muestran participaciones mayores que a precios de mercado, ya que el cálculo a precios de mercado incluye los impuestos menos subsidios sobre los productos y la producción en la contabilización del PIB.
}

que la información del SCN llega hasta 2005, la razón de participación salarial fue actualizada utilizando la evolución del índice medio de salario nominal, la tasa de empleo y el PIB a costo de factores.

Si se considera el último año con información disponible (alrededor de 2009), la participación de la masa salarial varía de 24\% en el Perú a 56,7\% en Costa Rica (véase el cuadro 1). El análisis de la evolución de esta participación indica que en la mayoría de los países se produce una caída ( 8 de un total de 12), con excepción de Chile, Costa Rica, el Paraguay y Venezuela (República Bolivariana de). De los países que evidencian una mejora en el período considerado, el caso de Costa Rica es el más diferenciado, ya que es el único en que se detecta una tendencia creciente y sostenida de la participación de los salarios en el PIB. En Chile, el Paraguay y Venezuela (República Bolivariana de), luego de un incremento importante entre 1990 y 2000, la participación de la masa salarial en el PIB declina en la última década ${ }^{14}$.

\footnotetext{
14 Los años específicos a que corresponden los datos del cuadro 1 para cada país se presentan en el cuadro A.3 del anexo. Las diferencias obedecen a la disponibilidad de datos.
} 
Los países que denotan un retroceso de la participación asalariada entre 1990 y fines de la década de 2000 tienen una trayectoria más heterogénea. En la Argentina y el Brasil se advierte una merma en los años noventa y una recuperación parcial en el decenio de 2000; en Bolivia (Estado Plurinacional de) mejora la participación asalariada en los años noventa y desciende en los años dos mil; Colombia, Honduras, Panamá y el Perú registran menguas a lo largo de todo el período (en los tres primeros casos con reducciones bastante acentuadas en los años noventa). México, que casi no registra variación entre los extremos, muestra un alza y posterior descenso prácticamente simétricos en las décadas de 1990 y 2000, respectivamente. La evolución en todos los años, respecto de los diferentes países, se presenta en el gráfico A.1 del anexo.

Participación de la masa salarial en el PIB a precio de factores

\begin{tabular}{|c|c|c|c|c|c|c|}
\hline & \multicolumn{3}{|c|}{ Participación de la masa salarial en el PIB } & \multicolumn{3}{|c|}{$\begin{array}{c}\text { Variación de la participación de la masa } \\
\text { salarial en el PIB }\end{array}$} \\
\hline & $\begin{array}{l}\text { Alrededor de } \\
1990 \text { (a) }\end{array}$ & $\begin{array}{l}\text { Alrededor de } \\
2000 \text { (b) }\end{array}$ & $\begin{array}{l}\text { Alrededor de } \\
2009 \text { (c) }\end{array}$ & $\begin{array}{l}\text { 1990-2000 } \\
\text { (b)-(a) }\end{array}$ & $\begin{array}{l}\text { 2000-2009 } \\
\text { (c)-(b) }\end{array}$ & $\begin{array}{l}\text { 1990-2009 } \\
\text { (c)-(a) }\end{array}$ \\
\hline Argentina & 44,7 & 40,5 & 42,9 & $-4,2$ & 2,4 & $-1,8$ \\
\hline Bolivia (Estado Plurinacional de) & 38,2 & 41,9 & 34,5 & 3,8 & $-7,4$ & $-3,6$ \\
\hline Brasil & 53,5 & 47,1 & 51,4 & $-6,3$ & 4,3 & $-2,1$ \\
\hline Chile & 38,7 & 46,5 & 45,4 & 7,8 & $-1,1$ & 6,7 \\
\hline Colombia & 41,4 & 36,2 & 36,1 & $-5,3$ & $-0,1$ & $-5,3$ \\
\hline Costa Rica & 48,3 & 50,6 & 56,7 & 2,3 & 6,1 & 8,4 \\
\hline Guatemala & & 36,3 & 32,8 & & $-3,5$ & \\
\hline Honduras & 54,8 & 47,5 & 47,4 & $-7,3$ & $-0,1$ & $-7,4$ \\
\hline México & 32,3 & 34,5 & 32,2 & 2,2 & $-2,3$ & $-0,1$ \\
\hline Nicaragua & 59,6 & 56,2 & & $-3,4$ & & \\
\hline Panamá & 58,6 & 40,6 & 35,2 & $-18,0$ & $-5,4$ & $-23,4$ \\
\hline Paraguay* & 43,4 & 59,0 & 47,2 & 15,7 & $-11,9$ & 3,8 \\
\hline Perú & 28,7 & 27,0 & 24,0 & $-1,8$ & $-3,0$ & $-4,7$ \\
\hline Uruguay & & 47,4 & 45,8 & & $-1,6$ & \\
\hline Venezuela (República Bolivariana de) & 31,1 & 35,6 & 33,5 & 4,5 & $-2,1$ & 2,4 \\
\hline
\end{tabular}

Fuente: elaboración propia sobre la base de información de CEPALSTAT, del Instituto Nacional de Estadística y Censos (INDEC) de la Argentina, y del Banco Central de Costa Rica, Banco de Guatemala y Banco Central del Uruguay.

* En el Paraguay, el dato correspondiente a 1990 es 50\% inferior al promedio del indicador en 1991-2009. Su consideración distorsiona enormemente la evolución de la serie temporal, por eso se optó por comenzar el análisis a partir de 1991 en este país. PIB: producto interno bruto.

Si se clasifican los países en tres grupos, distinguiendo a i) los que presentan una participación de la masa laboral de hasta $35 \%$, ii) aquellos en que esta se ubica entre $35 \%$ y $45 \%$ y, iii) aquellos en que rebasa el $45 \%$, el ordenamiento entre países resulta relativamente estable. En los tres años, el Perú y México se ubican siempre en el primer grupo, la Argentina, Colombia y el Paraguay en el segundo, y el Brasil, Costa Rica y Honduras en el tercero. El resto de los países no tienen información para los tres momentos, o cambian su clasificación en alguno de los tres momentos analizados (véase el cuadro A.4).

La evolución de la participación asalariada en el ingreso depende de las diferencias en la variación de los salarios reales y la productividad laboral. En el gráfico A.2 del anexo se representa la variación comparada de los salarios reales y la productividad laboral en los países de la región. En los años noventa, la productividad crece por sobre los salarios reales en la Argentina, Bolivia (Estado Plurinacional de), Chile, Guatemala, Panamá, el Perú, el Uruguay y Venezuela (República Bolivariana de) -lo que explica el retroceso de la participación asalariada en dicho período- , y por debajo en los casos de Colombia, Costa Rica y el Paraguay, lo que explica su aumento. En el Brasil, México y Nicaragua evolucionan a un ritmo semejante, por lo que no se observan cambios significativos en la distribución funcional del ingreso en esos países durante ese período. En los años dos mil los salarios reales crecen más rápidamente que la productividad laboral en la Argentina, el Brasil, Colombia, Costa Rica y Venezuela (República Bolivariana de), lo que explica el incremento de la participación asalariada en ese período. Lo contrario ocurre en Bolivia (Estado Plurinacional de), Chile, Guatemala, México, Panamá, el Paraguay, el Perú y el Uruguay. 
Un cambio muy relevante en los indicadores sociales de la región es la modificación en la tendencia a la profundización de la desigualdad personal del ingreso en los países latinoamericanos, que se advertía con mayor o menor intensidad en prácticamente todos ellos durante los años noventa. Luego de una década de crecimiento en la mayor parte de estos países durante aquellos años, a partir de 2002 o 2003, dependiendo del país, se aprecia un descenso en los indicadores de desigualdad en gran parte de los países de la región (véase CEPAL, 2012a y 2012b). Los diversos estudios coinciden en señalar el papel del mercado laboral en este descenso global de la desigualdad del ingreso de los hogares, ya que el ingreso derivado del mercado de trabajo se vuelve menos desigual y es la fuerza impulsora de la merma de la desigualdad ${ }^{15}$. Sin embargo, como

15 Una de las principales limitaciones de las encuestas de hogares consiste en sus problemas para capturar adecuadamente los ingresos de la parte alta de la distribución. Una alternativa que ha ganado importancia en los últimos años es la incorporación en el análisis de otras fuentes de datos, especialmente de los datos sobre ingresos y riqueza provenientes de los registros fiscales (veáse Piketty, 2003; Atkinson y Piketty, 2007 y 2010). Este tipo de estudios se han realizado para algunos países de la región (véanse Alvaredo, 2010; Alvaredo y Londoño, 2013; Burdín, Vigorito y Esponda, 2014). También se han corregido las estimaciones tradicionales de desigualdad de encuestas de hogares utilizando datos de cuentas nacionales (veáse Yamada, Castro y Bacigalupo, 2012). se evidencia a partir del análisis anterior y se ilustra en el gráfico 3, la mayor homogeneidad de los ingresos laborales, que se produjo en un contexto de ingresos crecientes, no ha estado acompañada de una mayor participación de la masa salarial en el PIB total. En los países latinoamericanos, con excepción de Costa Rica, la desigualdad del ingreso disminuyó entre 2002 y 2009, mientras que solo en la Argentina, el Brasil, Costa Rica y el Uruguay aumentó la participación de la masa salarial en el PIB total ${ }^{16}$. Las mejoras distributivas a nivel de los hogares no han significado, en general, un reparto más igualitario en términos de la apropiación del capital y el trabajo. Una hipótesis es que ello podría explicarse por el hecho de que, en la práctica, debido a cuestiones de captación, los relevamientos de ingresos en que se basan los indicadores de su distribución personal (como el coeficiente de Gini) no captan adecuadamente los ingresos derivados de la propiedad. Si esto fuera así, podría decirse que, en general, en la última década se advierte una distribución más equitativa de la masa salarial, sin cambios significativos (o incluso, una apropiación más concentrada en los sectores propietarios) en la distribución del excedente económico generado en la región.

16 En el gráfico 3 no se incluye a Guatemala, ya que en este caso el último dato disponible sobre el índice de Gini corresponde a 2006.

GRÁFICO 3

Variación de la participación de los salarios en el PIB y del índice de Gini, 2002-2009
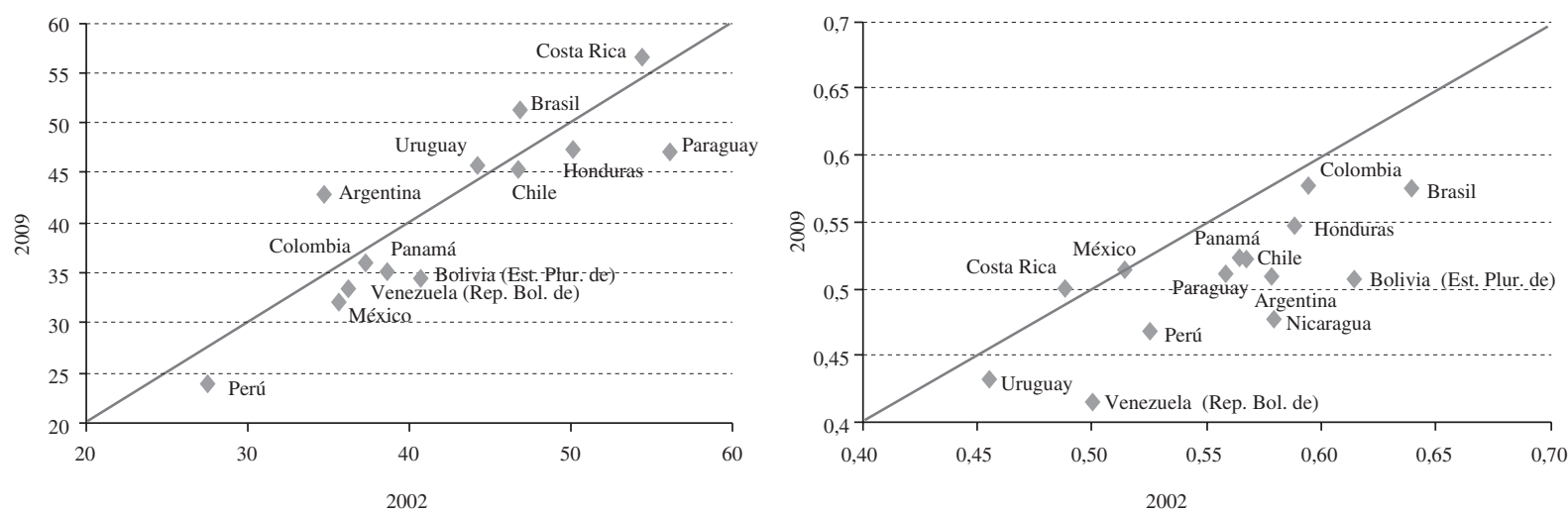

Fuente: elaboración propia sobre la base de información de CEPALSTAT, del Instituto Nacional de Estadística y Censos (INDEC) de la Argentina y del Banco Central de Costa Rica y Banco Central del Uruguay.

PIB: producto interno bruto. 


\section{VII \\ Importancia del trabajo independiente en América Latina}

Una de las características distintivas de los mercados laborales de América Latina es el alto peso del trabajo independiente, que comprende a trabajadores por cuenta propia y patrones. El trabajo independiente concentra una proporción muy relevante del empleo total en la región (casi el 32\% respecto de los países considerados). Su participación en el empleo total varía del $22 \%$ en la
Argentina a más del 49\% en Colombia, de acuerdo con la última información disponible (alrededor de 2011). En la última década se ha incrementado la importancia del empleo asalariado (pasando del 59,8\% al 63,7\% del total de ocupados en la región), y se produce también un leve aumento en el peso de los ingresos salariales en el ingreso per cápita de los hogares (véase el cuadro 2).

Trabajo e ingresos asalariados e independientes

\begin{tabular}{|c|c|c|c|c|c|c|c|c|}
\hline & \multicolumn{4}{|c|}{ Alrededor de 2000} & \multicolumn{4}{|c|}{ Alrededor de 2011} \\
\hline & \multicolumn{2}{|c|}{$\begin{array}{l}\text { Participación en el total } \\
\text { de ocupados }\end{array}$} & \multicolumn{2}{|c|}{$\begin{array}{c}\text { Participación en el } \\
\text { ingreso de los hogares }\end{array}$} & \multicolumn{2}{|c|}{$\begin{array}{l}\text { Participación en el total } \\
\text { de ocupados }\end{array}$} & \multicolumn{2}{|c|}{$\begin{array}{l}\text { Participación en el } \\
\text { ingreso de los hogares }\end{array}$} \\
\hline & Asalariados & Independientes & $\begin{array}{l}\text { Ingresos } \\
\text { salariales }\end{array}$ & $\begin{array}{c}\text { Ingresos } \\
\text { independientes }\end{array}$ & Asalariados & Independientes & $\begin{array}{l}\text { Ingresos } \\
\text { salariales }\end{array}$ & $\begin{array}{c}\text { Ingresos } \\
\text { independientes }\end{array}$ \\
\hline $\begin{array}{l}\text { Argentina } \\
\text { Bolivia (Estado }\end{array}$ & 72,0 & 26,8 & 42,4 & 30,3 & 76,9 & 22,4 & 49,8 & 25,1 \\
\hline Plurinacional de) & 32,1 & 47,5 & 41,5 & 27,7 & 41,3 & 40,8 & 46,8 & 37,3 \\
\hline Brasil & 62,8 & 31,1 & 35,4 & 20,7 & 68,4 & 28,7 & 42,4 & 19,4 \\
\hline Chile & 74,4 & 24,1 & 46,4 & 29,9 & 77,4 & 22,3 & 52,8 & 26,3 \\
\hline Colombia & 49,4 & 45,6 & 45,2 & 27,8 & 46,0 & 49,5 & 44,0 & 29,7 \\
\hline Costa Rica & 71,6 & 26,5 & 64,9 & 19,8 & 75,9 & 22,8 & 62,8 & 17,3 \\
\hline Guatemala & 47,0 & 38,8 & 38,2 & 45,8 & 51,4 & 35,0 & 32,5 & 42,1 \\
\hline Honduras & 49,6 & 41,2 & 45,5 & 29,0 & 43,7 & 45,7 & 47,4 & 28,5 \\
\hline México & 66,1 & 26,6 & 46,7 & 28,4 & 73,0 & 22,1 & 49,7 & 13,6 \\
\hline Nicaragua & 52,3 & 35,1 & 50,7 & 40,8 & 48,9 & 39,3 & 50,4 & 38,2 \\
\hline Panamá & 62,7 & 32,3 & 60,3 & 24,5 & 67,5 & 28,7 & 54,5 & 28,9 \\
\hline Perú & 40,5 & 44,0 & 39,4 & 29,8 & 44,9 & 42,9 & 42,5 & 31,1 \\
\hline Paraguay & 44,8 & 45,2 & 42,9 & 37,8 & 52,9 & 39,5 & 47,0 & 41,7 \\
\hline Uruguay & 72,7 & 25,7 & 42,1 & 16,1 & 71,9 & 26,8 & 46,5 & 16,3 \\
\hline $\begin{array}{l}\text { Venezuela } \\
\text { (República }\end{array}$ & & & & & & & & \\
\hline Bolivariana de) & 56,4 & 41,9 & 45,5 & 39,1 & 57,2 & 41,9 & 52,3 & 27,9 \\
\hline América Latina & 59,8 & 33,4 & 45,4 & 31,7 & 63,7 & 31,6 & 46,2 & 30,9 \\
\hline
\end{tabular}

Fuente: Comisión Económica para América Latina y el Caribe (CEPAL), sobre la base de datos de encuestas continuas de hogares.

Los ingresos reportados por los trabajadores independientes representan una proporción muy relevante del ingreso per cápita de los hogares (alrededor del $31 \%$ en la región). Teóricamente, debido a la naturaleza de estas actividades, una parte de esos ingresos son retribuciones al trabajo y otra parte son retribuciones al capital. Como fuera explicado anteriormente, en la participación de la masa salarial que surge de las cuentas nacionales no se incorporan estos ingresos, lo que constituye una importante limitación, sobre todo a la hora de realizar comparaciones entre países con diversos grados de desarrollo. A continuación se presentan dos ajustes a las estimaciones tradicionales de participación del ingreso laboral, con los que se procura incorporar los ingresos de los trabajadores independientes. 


\section{VIII}

\section{Reestimación de la participación salarial incluido el ingreso del trabajo independiente}

Como fuera detallado en la subsección V.2, la estimación de la participación de los ingresos laborales en el PIB se realizó sobre la base de dos ajustes. El primero es el más extendido en la literatura (similar, por ejemplo, al utilizado en OIT, 2013), y consiste en imputar el salario promedio estimado a partir de los datos del SCN a todos los trabajadores independientes, y adicionar ese monto a los sueldos y salario del $\mathrm{SCN}^{17}$.

La segunda opción, más rigurosa, y que fuera detallada en la subsección V.2, consiste en utilizar la información de las encuestas continuas de hogares para estimar la relación entre la masa de ingresos salariales y la masa de ingresos por trabajo de los trabajadores independientes, y sobre la base de esta relación corregir la participación de la masa salarial en el PIB.

Se estimaron ecuaciones salariales para el conjunto de asalariados de la economía, incluidos como variables dependientes el sexo, la edad y su expresión cuadrática, los años de escolaridad, y variables binarias que distinguen por rama de actividad ${ }^{18}$. A partir de los coeficientes estimados en estas ecuaciones, se realizó una predicción de ingresos laborales para cada uno de los trabajadores independientes (tanto trabajadores por cuenta propia como patrones) incluidos en las encuestas de hogares. Cuando estas predicciones de ingreso laboral de los trabajadores independientes resultaron inferiores al ingreso que estos reportan en las encuestas de hogares, se asumió que esa diferencia corresponde a la retribución al capital. Por lo tanto, se considera como ingreso laboral de esos trabajadores el que resulta de la predicción. En el caso en que el ingreso predicho supera al ingreso declarado en las encuestas de hogares, se toma todo el ingreso declarado en las encuestas de hogares como ingreso laboral de los trabajadores independientes. La adopción de este criterio implicó utilizar las predicciones solo en el $41 \%$ de los trabajadores por cuenta propia (promedio entre países y para los años considerados), aunque entre

\footnotetext{
17 En concreto, se adiciona el producto del salario promedio por la cantidad de trabajadores independientes del país. Las estimaciones del total de trabajadores independientes surgen de las encuestas continuas de hogares y de CEPALSTAT.

18 Los resultados de estas estimaciones están disponibles para quienes deseen solicitarlos a los autores.
}

los patrones las predicciones fueron utilizadas para el $71 \%$. Tal resultado resulta razonable, ya que estaría reflejando la mayor importancia de las retribuciones al capital entre los patrones. Se estima así un nuevo vector de ingresos laborales para cada uno de los trabajadores independientes incluido en la encuesta de hogares. Se calcula luego la relación entre la masa salarial reportada por la encuesta y la masa de ingresos laborales estimada con esta metodología. Esa razón es aplicada a la masa salarial reportada en el SCN, para cada país y año, y de esta manera se estima la masa de ingresos laborales totales (es decir, ingresos salariales más ingresos laborales de los trabajadores independientes) que se coteja con el PIB. En el cuadro 3 se comparan los tres resultados: el peso de la masa salarial en el PIB según el SCN, la corrección considerando que los trabajadores independientes perciben un ingreso similar al salario promedio y la corrección más detallada con la metodología antes explicada.

En todos los casos, el ajuste a través de la imputación de salarios medios produce una sobreestimación importante de la masa de ingresos laborales en los países de la región. En los casos de Bolivia (Estado Plurinacional de) (2000), Honduras (2010) y el Paraguay (2001), esta corrección implica que casi la totalidad del PIB medido a costo de factores correspondería a ingresos laborales. Cuando se realizan las estimaciones por el segundo método, se aprecia que una proporción relevante de los trabajadores independientes, especialmente en el caso de los trabajadores por cuenta propia, tienen ingresos declarados en las encuestas que son inferiores a los que surgen de las predicciones de acuerdo con sus características personales y rama de actividad ${ }^{19}$. Este diferencial de ingresos con respecto a los trabajadores asalariados indica que la simple imputación de los salarios promedio implica un sesgo relevante. En promedio, la importancia de los ingresos salariales se incrementa en 25 puntos con esta segunda estimación.

\footnotetext{
19 La metodología de ajuste que se utiliza en este trabajo presenta la limitación de asumir que las contribuciones a la seguridad social e impuestos son similares entre trabajadores asalariados e independientes, y que los porcentajes de cotizantes son similares también - ya que se aplica una relación obtenida a partir de ingresos líquidos de las encuestas de hogares (en la mayor parte de los países) — a una variable (masa salarial) que incluye las contribuciones.
} 
CUADRO 3

Participación de la masa salarial y del ingreso laboral estimado en el PIB

\begin{tabular}{|c|c|c|c|c|}
\hline & & Salarios/PIB & $\begin{array}{l}\text { Ingresos laborales/PIB } \\
\text { Estimación } 1\end{array}$ & $\begin{array}{l}\text { Ingresos laborales/PIB } \\
\text { Estimación } 2\end{array}$ \\
\hline \multirow[t]{2}{*}{ Argentina } & 2000 & 40,5 & 54,4 & 45,7 \\
\hline & 2006 & 41,5 & 54,2 & 48,7 \\
\hline \multirow[t]{2}{*}{ Bolivia (Estado Plurinacional de) } & 2000 & 41,9 & 100,1 & 56,9 \\
\hline & 2007 & 34,5 & 70,9 & 47,0 \\
\hline \multirow[t]{2}{*}{ Brasil } & 2001 & 47,7 & 68,2 & 56,9 \\
\hline & 2009 & 51,4 & 70,6 & 59,7 \\
\hline \multirow[t]{2}{*}{ Chile } & 2000 & 46,5 & 61,3 & 54,9 \\
\hline & 2009 & 44,5 & 58,1 & 52,8 \\
\hline \multirow[t]{2}{*}{ Colombia } & 2000 & 36,2 & 68,3 & 41,2 \\
\hline & 2009 & 44,5 & 58,1 & 52,8 \\
\hline \multirow{2}{*}{ Costa Rica } & 2000 & 50,6 & 69,8 & 55,8 \\
\hline & 2010 & 56,9 & 73,8 & 65,0 \\
\hline \multirow[t]{2}{*}{ Guatemala } & 2003 & 35,5 & 61,3 & 46,6 \\
\hline & 2007 & 33,5 & 53,7 & 44,4 \\
\hline Honduras & 2010 & 47,5 & 93,1 & 65,5 \\
\hline \multirow[t]{2}{*}{ México } & 2000 & 34,5 & 48,2 & 45,6 \\
\hline & 2008 & 31,4 & 41,1 & 36,2 \\
\hline \multirow[t]{2}{*}{ Panamá } & 2000 & 40,6 & 54,4 & 47,8 \\
\hline & 2009 & 35,2 & 49,2 & 40,6 \\
\hline \multirow[t]{2}{*}{ Paraguay } & 2001 & 58,5 & 116,7 & 84,3 \\
\hline & 2007 & 47,2 & 87,7 & 68,0 \\
\hline \multirow[t]{2}{*}{ Perú } & 2000 & 27,0 & 55,1 & 35,9 \\
\hline & 2009 & 23,3 & 46,0 & 31,3 \\
\hline \multirow[t]{2}{*}{ Uruguay } & 2000 & 47,4 & 63,6 & 57,0 \\
\hline & 2010 & 45,8 & 62,0 & 54,3 \\
\hline \multirow[t]{2}{*}{ Venezuela (República Bolivariana de) } & 2000 & 35,6 & 56,4 & 46,5 \\
\hline & 2010 & 38,4 & 58,2 & 44,5 \\
\hline
\end{tabular}

Fuente: elaboración propia sobre la base de información de CEPALSTAT, del Instituto Nacional de Estadística y Censos (INDEC) de la Argentina, el Banco Central de Costa Rica, el Banco Central del Uruguay y encuestas continuas de hogares.

PIB: producto interno bruto.

La estimación a través del segundo método redunda en un incremento considerable en la participación de los ingresos laborales cuando se la compara con los datos del SCN, que solo incluyen la masa salarial, pero los resultados son inferiores a los derivados de la imputación del salario promedio. En esta nueva estimación, que se considera que es la que mejor en este trabajo, se refleja la importancia de los ingresos laborales, que abarcan un rango que va del $31 \%$ del PIB en el Perú al 65\% en Costa Rica. En promedio para todos los países considerados, la importancia de los ingresos laborales aumenta 10 puntos en relación con la que surge al considerar tan solo los ingresos salariales.

Las variaciones de la masa salarial son relativamente similares, aunque se ven acentuadas en el caso de los países con mayor relevancia del trabajo independiente (por ejemplo, Colombia).
La información de la encuesta de hogares permite analizar la distribución de la masa total de ingresos laborales estimados con la segunda metodología, según características de los trabajadores. Se analiza la distribución de estos ingresos, resultantes de la suma de ingresos salariales y nuevo vector de ingresos laborales de los trabajadores por cuenta propia en la encuesta de hogares, según nivel educativo de los trabajadores y sexo. En relación con el nivel educativo, tal distribución muestra una gran estabilidad entre países; más de la mitad de la masa de ingresos laborales es generada por los trabajadores con educación terciaria (completa e incompleta) (véase el gráfico 4). Las mujeres, a su vez, generan en promedio el 35\% de los ingresos laborales de las economías de la región, y su participación ha sido creciente en la mayoría de los países analizados (véase el gráfico 5). 
GRÁFICO 4

Porcentaje del ingreso laboral total según nivel educacional, alrededor de 2000-2009a

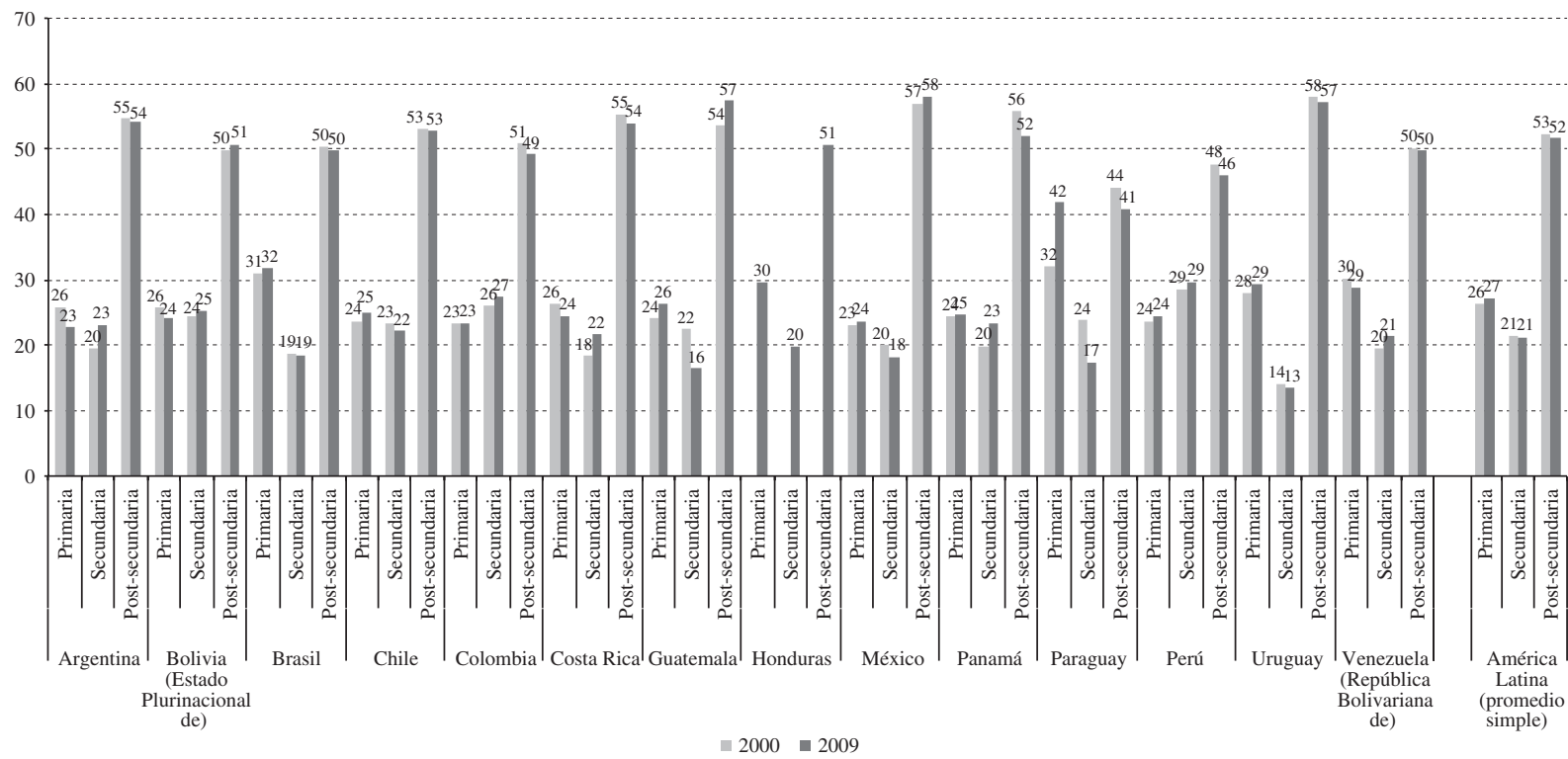

Fuente: elaboración propia, sobre la base de datos de encuestas continuas de hogares.

a Las barras refieren al porcentaje del ingreso laboral total que tiene el nivel educacional finalizado (primaria, secundaria y postsecundaria). Para el primer período, los años distintos a 2000 son 2001 para el Brasil y 2003 para Guatemala. Para el segundo período, son 2006 para la Argentina, 2007 para Bolivia (Estado Plurinacional de), 2008 para México y 2010 para Costa Rica, Guatemala y el Uruguay.

GRÁFICO 5

Porcentaje del ingreso laboral total generado por mujeres, alrededor de 2000-2009a

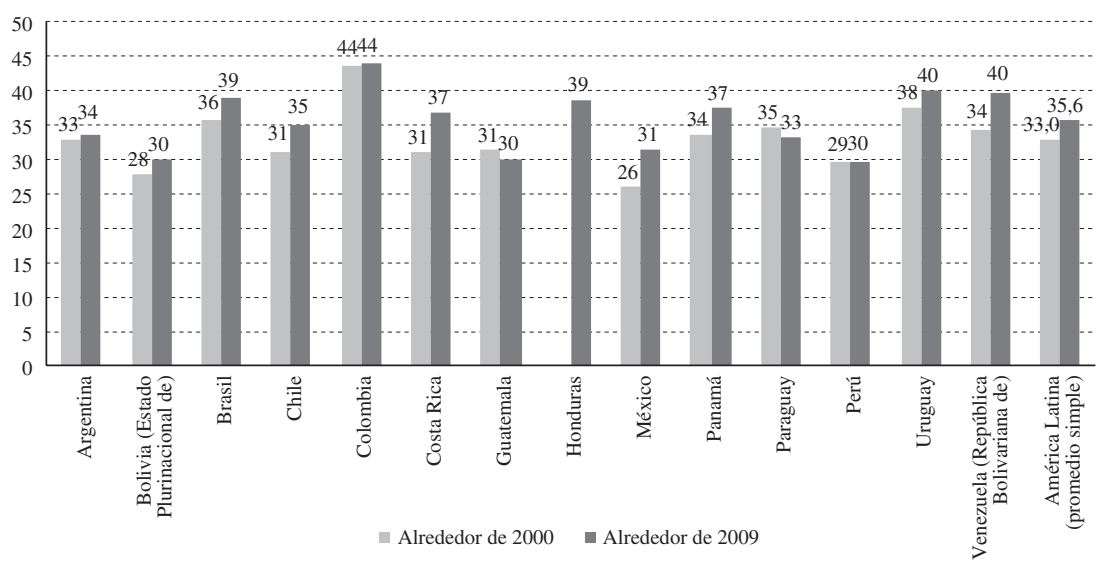

Fuente: elaboración propia, sobre la base de datos de encuestas continuas de hogares.

a Las barras refieren al porcentaje del ingreso laboral total que tiene el nivel educacional finalizado (primaria, secundaria y postsecundaria). Para el primer período, los años distintos a 2000 son 2001 para el Brasil y 2003 para Guatemala. Para el segundo período, son 2006 para la Argentina, 2007 para Bolivia (Estado Plurinacional de), 2008 para México y 2010 para Costa Rica, Guatemala y el Uruguay. 


\section{IX}

\section{Comentarios finales}

El análisis actual sobre la distribución del ingreso se centra en la desigualdad entre hogares y personas, profundizando en los fundamentos microeconómicos de su evolución. En este artículo se ha argumentado que resulta relevante mantener la perspectiva funcional y procurar comprender la realidad considerando y relacionando ambos enfoques. Para la región, la integración de la distribución funcional del ingreso a las agendas de investigación constituye un desafío, debido en parte a las limitaciones de la información disponible. Sin embargo, tal integración puede mostrarnos nuevas aristas sobre la situación distributiva regional. La caída en la desigualdad del ingreso entre los hogares, que la región experimenta desde hace una década, no ha implicado mejoras en términos de la apropiación de los frutos del crecimiento por parte de los trabajadores.

ANEXO

CUADRO A.1

Disponibilidad de información en CEPAL

\begin{tabular}{|c|c|c|c|c|c|c|c|}
\hline País & Período & RL & $\mathrm{EE}$ & CKF & PIBcf & $(\mathrm{T}-\mathrm{S}) \mathrm{xm}$ & PIBpm \\
\hline Bolivia (Estado Plurinacional de) & $1988 / 2008$ & & & & & & \\
\hline Brasil & $\begin{array}{l}1970-1975-1980-1985^{a} \\
1990 / 2009\end{array}$ & & & & & & \\
\hline Chile & $\begin{array}{l}1960 / 1985 \\
1985 / 1996 \\
1996 / 2006 \\
2003 / 2009 \\
2008 / 2010\end{array}$ & & & & & & \\
\hline Colombia & $\begin{array}{l}1970 / 1994 \\
1994 / 2000 \\
2000 / 2010\end{array}$ & & & & & & \\
\hline Costa Rica & 1970/1991 & & & & & & \\
\hline Ecuador & $1970 / 1989$ & & & & & & \\
\hline Honduras & $\begin{array}{l}1950 / 1995 \\
1996 / 2000 \\
2000 / 2011 \\
\end{array}$ & & & & & & \\
\hline México & $\begin{array}{l}1970 / 1980 \\
1980 / 1988 \\
1988 / 2003 \\
2003 / 2011\end{array}$ & & & & & & \\
\hline Nicaragua & $1994 / 2011$ & & & & & & \\
\hline Panamá & $\begin{array}{l}1960 / 1970 \\
1970 / 1980 \\
1980 / 1996 \\
1996 / 2011\end{array}$ & & & & & & \\
\hline Paraguay & $\begin{array}{l}1970 / 1991 \\
1991 / 2007 \\
\end{array}$ & & & & & & \\
\hline Perú & $1991 / 2010$ & & & & & & \\
\hline Uruguay & $\begin{array}{l}1971 / 1983 \\
1983 / 1988\end{array}$ & & & & & & \\
\hline Venezuela (República Bolivariana de) & $\begin{array}{l}1970 / 1984 \\
1984 / 1997 \\
1997 / 2011^{\mathrm{b}}\end{array}$ & & & & & & \\
\hline
\end{tabular}

Fuente: elaboración propia sobre la base de información de CEPALSTAT.

a El guión no denota un período de años, sino únicamente que existe información para esos años en particular.

b No se dispone de datos para el año 2010.

Nota: RL: remuneración a los asalariados; EE: excedente de explotación; CKF: consumo de capital fijo; PIBcf: producto interno bruto a costo de factores; (T-S)xm: impuestos netos de subsidios a la producción e importaciones; PIBpm: producto interno bruto a precios de mercado. 
CUADRO A.2

Disponibilidad de información en UNSD

\begin{tabular}{|c|c|c|c|c|c|c|c|}
\hline País & Período & RL & IM & $\mathrm{EE}$ & CKF & $(\mathrm{T}-\mathrm{S}) \mathrm{xm}$ & VABpb \\
\hline Argentina & $1993 / 2007$ & & & & & & \\
\hline Bolivia (Estado Plurinacional de) & $1970 / 2011$ & & & & & & \\
\hline Brasil & $\begin{array}{l}1992 / 2003 \\
1995 / 2009\end{array}$ & & & & & & \\
\hline Chile & $\begin{array}{l}1974 / 1985 \\
1985 / 1998 \\
1996 / 2009 \\
2008 / 2010\end{array}$ & & & & & & \\
\hline Colombia & $\begin{array}{l}1970 / 1995 \\
1992 / 2005 \\
2000 / 2010\end{array}$ & & & & & & \\
\hline Costa Rica & $\begin{array}{l}1970 / 1993 \\
1991 / 2010 \\
\end{array}$ & & & & & & \\
\hline Ecuador & $1970 / 1991$ & & & & & & \\
\hline Guatemala & $2001 / 2010$ & & & & & & \\
\hline Honduras & $\begin{array}{l}1992 / 2006 \\
2000 / 2010\end{array}$ & & & & & & \\
\hline México & $\begin{array}{l}1988 / 2004 \\
1993 / 2004 \\
2003 / 2010\end{array}$ & & & & & & \\
\hline Nicaragua & $1994 / 2007$ & & & & & & \\
\hline Panamá & $\begin{array}{l}1989 / 2000 \\
1996 / 2010\end{array}$ & & & & & & \\
\hline Paraguay & $1994 / 2010$ & & & & & & \\
\hline Perú & $\begin{array}{l}1970 / 1998 \\
1991 / 2010 \\
\end{array}$ & & & & & & \\
\hline República Dominicana & $1991 / 2005$ & & & & & & \\
\hline Uruguay & $1997 / 2005$ & & & & & & \\
\hline Venezuela (República Bolivariana de) & $\begin{array}{l}1970 / 1984 \\
1984 / 2002 \\
1997 / 2010\end{array}$ & & & & & & \\
\hline
\end{tabular}

Fuente: elaboración propia sobre la base de datos de la División de Estadística de las Naciones Unidas (UNSD).

Nota: RL: remuneración a los asalariados; IM: ingreso mixto; EE: excedente de explotación; CKF: consumo de capital fijo: (T-S)xm: impuestos netos de subsidios a la producción e importaciones; VABpb: valor agregado bruto a precios básicos. 
Años correspondientes por país para datos del cuadro 1

\begin{tabular}{|c|c|c|c|}
\hline & Alrededor de 1990 & Alrededor de 2000 & Alrededor de 2009 \\
\hline Argentina & 1993 & 2000 & 2007 \\
\hline Bolivia (Estado Plurinacional de) & 1990 & 2000 & 2007 \\
\hline Brasil & 1991 & 2000 & 2009 \\
\hline Chile & 1990 & 2000 & 2009 \\
\hline Colombia & 1990 & 2000 & 2009 \\
\hline Costa Rica & 1991 & 2000 & 2009 \\
\hline Guatemala & $\ldots$ & 2001 & 2009 \\
\hline Honduras & 1990 & 2000 & 2009 \\
\hline México & 1990 & 2000 & 2009 \\
\hline Nicaragua & 1994 & 2000 & n.d \\
\hline Panamá & 1990 & 2000 & 2009 \\
\hline Paraguay $^{\mathrm{a}}$ & 1991 & 2000 & 2009 \\
\hline Perú & 1990 & 2000 & 2009 \\
\hline Uruguay $^{b}$ & n.d & 2000 & 2009 \\
\hline Venezuela (República Bolivariana de) & 1990 & 2000 & 2009 \\
\hline
\end{tabular}

Fuente: elaboración propia.

a En el Paraguay, el dato correspondiente a 1990 es 50\% inferior al promedio del indicador en 1991-2009. Su consideración distorsiona enormemente la evolución de la serie temporal, por eso se optó por comenzar el análisis a partir de 1991 en este país.

b La información del Banco Central del Uruguay llega hasta 2005. La razón fue actualizada utilizando la evolución del índice medio de salario nominal, la tasa de empleo y el producto interno bruto (PIB) a costo de factores.

CUADRO A.4

Clasificación de los países de acuerdo con el valor de la participación de la masa salarial

\begin{tabular}{|c|c|c|c|}
\hline Salario/PIB & Alrededor de 1990 & Alrededor de 2000 & Alrededor de 2009 \\
\hline Hasta $35 \%$ & $\begin{array}{l}\text { Perú, México, Venezuela } \\
\text { (República Bolivariana de) }\end{array}$ & México, Perú & $\begin{array}{l}\text { Bolivia (Estado Plurinacional } \\
\text { de), Guatemala, México, } \\
\text { Perú, Venezuela (República } \\
\text { Bolivariana de) }\end{array}$ \\
\hline $35 \%-45 \%$ & $\begin{array}{l}\text { Argentina, Bolivia (Estado } \\
\text { Plurinacional de), Chile, } \\
\text { Colombia, Paraguay }\end{array}$ & $\begin{array}{l}\text { Guatemala, Argentina, Bolivia } \\
\text { (Estado Plurinacional de), } \\
\text { Colombia, Panamá, Venezuela } \\
\text { (República Bolivariana de) }\end{array}$ & Argentina, Colombia, Panamá \\
\hline Más del 45\% & $\begin{array}{l}\text { Brasil, Costa Rica, Honduras, } \\
\text { Nicaragua, Panamá }\end{array}$ & $\begin{array}{l}\text { Brasil, Chile, Costa Rica, } \\
\text { Honduras, Nicaragua, Paraguay, } \\
\text { Uruguay }\end{array}$ & $\begin{array}{l}\text { Brasil, Chile, Costa Rica, } \\
\text { Honduras, Paraguay, Uruguay }\end{array}$ \\
\hline
\end{tabular}

Fuente: elaboración propia.

PIB: producto interno bruto. 
GRÁFICO A.1

América Latina (15 países):

evolución de la participación de la masa salarial en el PIB

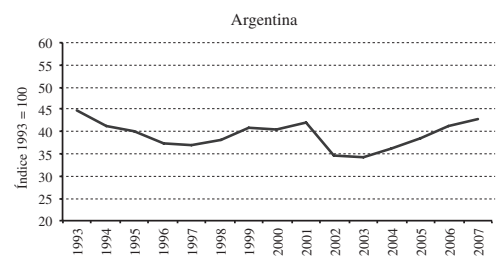

Chile

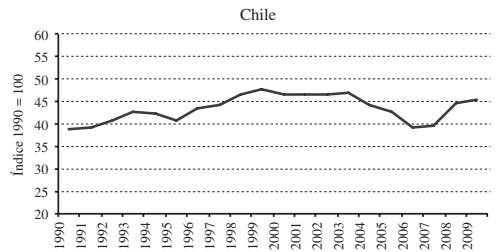

Guatemala

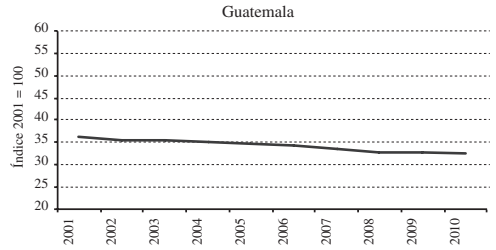

Nicaragu

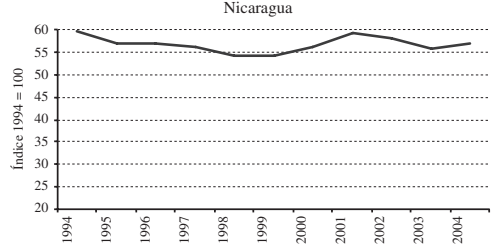

Peru

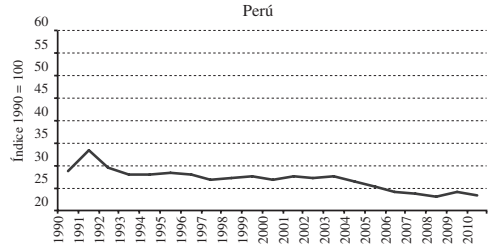

Bolivia (Estado Plurinacional de)

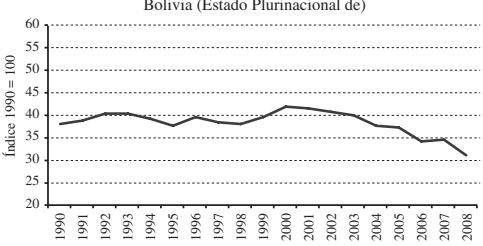

Colombia

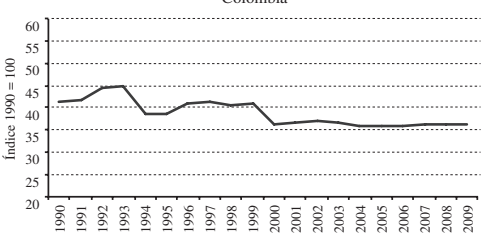

Honduras

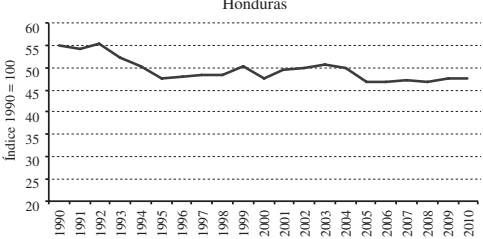

Panamá

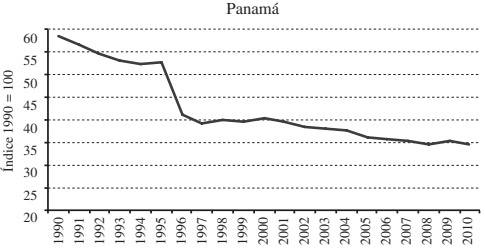

Uruguay

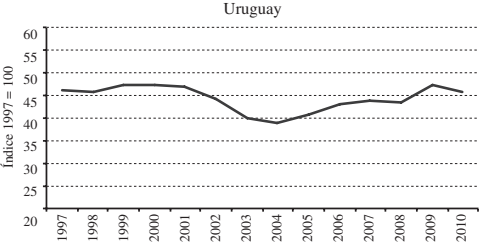

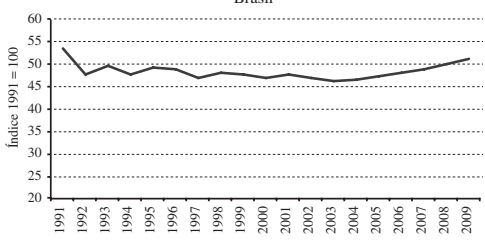

Costa Rica

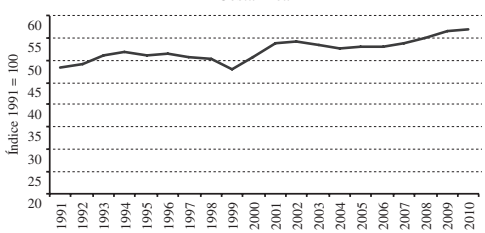

México

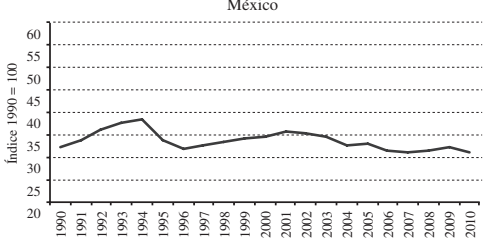

Paraguay

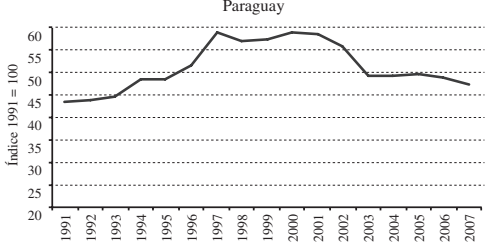

Venezuela (República Bolivariana de)

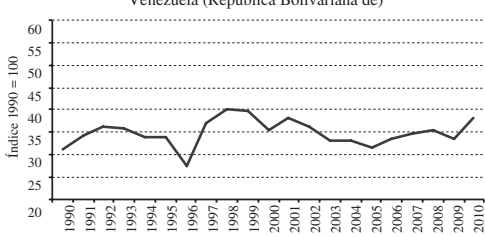

Fuente: elaboración propia, sobre la base de información de CEPALSTAT, del Instituto Nacional de Estadística y Censos (INDEC) de la Argentina y del Banco Central de Costa Rica, Banco de Guatemala y Banco Central del Uruguay.

PIB: producto interno bruto. 
GRÁFICO A.2

América Latina (14 países):

evolución de los salarios reales y la productividad del trabajo, 1990-2010

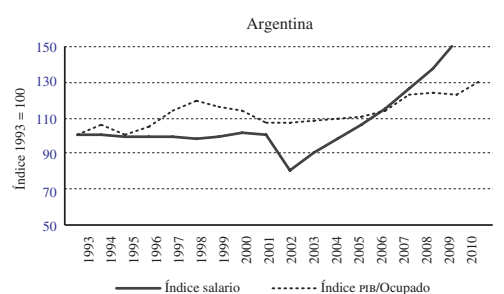

Chile

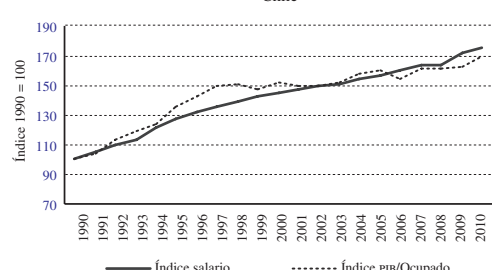

Guatemala

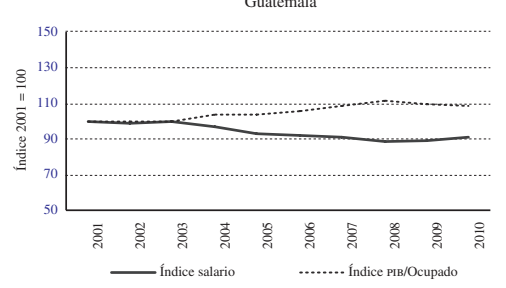

Panamá

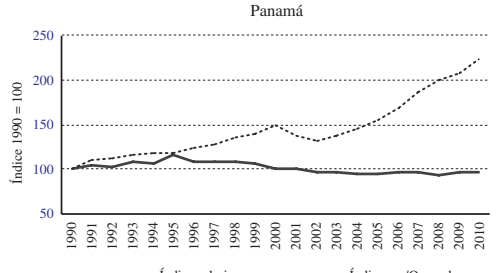

— Índice salario

Uruguay

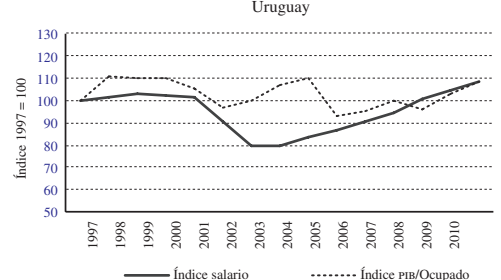

Bolivia (Estado Plurinacional de)

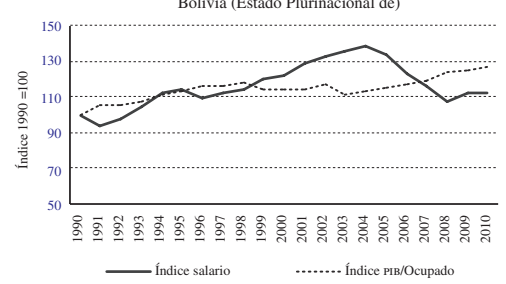

Colombia

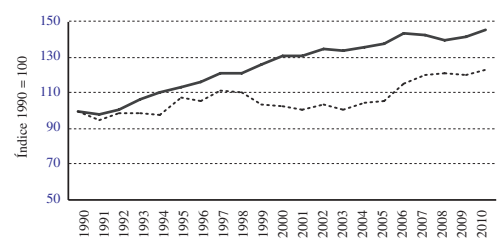

México

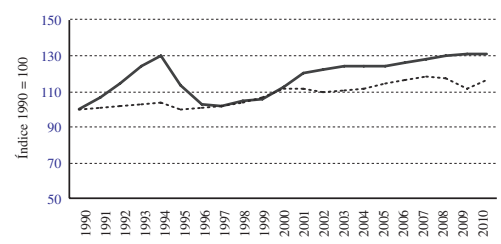

— Índice salario $\quad$......... Índice PIB/Ocupado

Paraguay

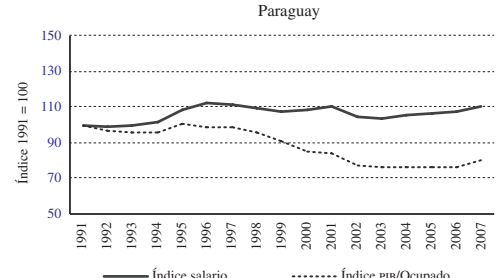

Venezuela (República Bolivariana de)

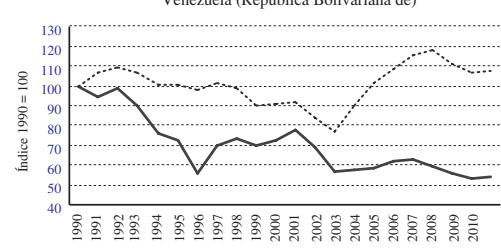

— Índice salario $\quad$......... Índice PII/Ocupado

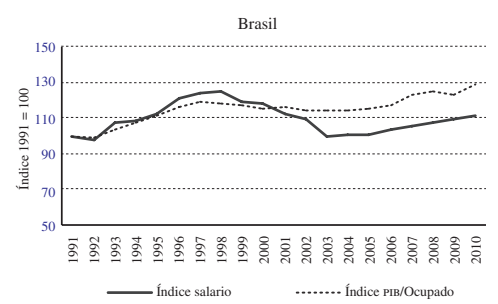

Costa Rica

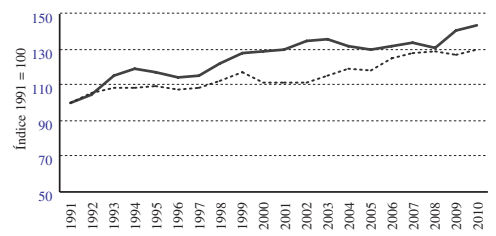

— Índice salario _........ Índice PII/Ocupado

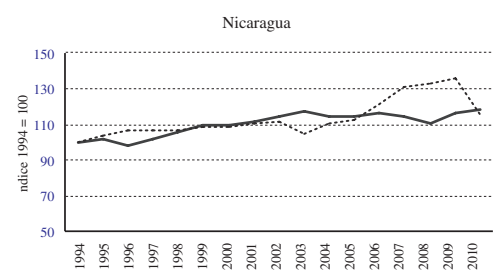

Perú

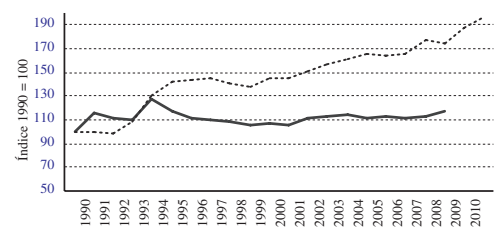

IIndice salario - ........ Índice PIB/Ocupado

Fuente: elaboración propia sobre la base de información de CEPALSTAT, del Instituto Nacional de Estadística y Censos (INDEC) de la Argentina y del Banco Central de Costa Rica, Banco de Guatemala y Banco Central del Uruguay.

PIB: producto interno bruto. 


\section{Bibliografía}

Abeles, M. y F. Toledo (2011), "Distribución del ingreso y análisis macroeconómico: Un repaso de la literatura y de los desafíos de política económica", Distribución del ingreso. Enfoques y políticas públicas desde el Sur, M. Novick y S. Villafañe (eds.), Buenos Aires, Programa de las Naciones Unidas para el Desarrollo (PNUD)/Ministerio de Trabajo, Empleo y Seguridad Social de Argentina.

Alvaredo, F. (2010), "The rich in Argentina over the twentieth century", Top Incomes: A Global Perspective, A. Atkinson y T. Piketty (eds.), Oxford University Press.

Alvaredo, F. y J. Londoño (2013), "High incomes and personal taxation in a developing economy: Colombia 1993-2010", inédito.

Amarante, V. y A. Vigorito (2011), "Los futuros posibles de la desigualdad de ingresos", La aventura uruguaya. ¿Naides más que naides?, R. Arocena y G. Caetano (eds.), Montevideo, Debate.

Antras, P. (2004), "Is the U.S. aggregate production function CobbDouglas? New estimates of the elasticity of substitution", Contributions in Macroeconomics, vol. 4, $\mathrm{N}^{\circ}$ 1, Universidad de Harvard.

Atkinson, A. (2009), "Factor shares: the principal problem of political economy?", Oxford Review of Economic Policy, vol. 25, $\mathrm{N}^{\circ} 1$, Oxford University Press.

Atkinson, A. y T. Piketty (2010), Top Incomes: A Global Perspective, Oxford University Press.

(2007), Top Incomes over the Twentieth Century: A Contrast between Continental European and English-Speaking Countries, Oxford University Press.

Bentolila, S. y G. Saint-Paul (2003), "Explaining movements in the labor share", The B.E. Journal of Macroeconomics, vol. 3, $\mathrm{N}^{\circ} 1$, De Gruyter.

Bernanke, B.S. y R.S. Gürkaynak (2002), "Is growth exogenous? Taking Mankiw, Romer, and Weil seriously", NBER Macroeconomics Annual 2001, vol. 16, Cambridge, Massachusetts, National Bureau of Economic Research.

Bhaduri, A. (1983), The Economic Structure of Backward Agriculture, Academic Press.

Bhaduri, A. y S. Marglin (1990), "Unemployment and the real wage: the economic basis for contesting political ideologies", Cambridge Journal of Economics, vol. 14, $\mathrm{N}^{\circ} 4$, Oxford University Press.

Burdín, G., A. Vigorito y F. Esponda (2014), "Desigualdad y sectores de altos ingresos en Uruguay: Un análisis en base a registros tributarios y encuestas de hogares para el período 2009-2011", Documento de Trabajo, $\mathrm{N}^{\circ}$ 06/04, Montevideo, Instituto de Economía, Universidad de la República.

Campodónico, H. (2008), "Renta petrolera y minera en países seleccionados de América Latina", Documento de Proyecto, $\mathrm{N}^{\circ} 188$ (LC/W.188), Santiago de Chile, Comisión Económica para América Latina y el Caribe (CEPAL).

CEPAL (Comisión Económica para América Latina y el Caribe) (2013), Recursos naturales en UNASUR. Situación y tendencias para una agenda de desarrollo regional (LC/L.3627), Santiago de Chile.

(2012a), Cambio estructural para la igualdad: Una visión integrada del desarrollo (LC/G.2524(SES.34/3)), Santiago de Chile.

(2012b), Panorama Social de América Latina 2011 (LC/G.2514-P), Santiago de Chile. Publicación de las Naciones Unidas, $\mathrm{N}^{\circ}$ de venta: S.12.II.G.6.

Comisión Europea (2007), The Labour Income Share in the European Union, Bruselas, Dirección General de Empleo, Asuntos Sociales e Igualdad de Oportunidades.

Daudey, E. y C. García-Peñalosa (2007), "The personal and the factor distributions of income in a cross-section of countries", The Journal of Development Studies, vol. 43, N ${ }^{\circ}$ 5, Taylor \& Francis.
Ellis, L. y K. Smith (2010), "The global upward trend in the profit share", Applied Economics Quarterly, vol. 56, № 3, Duncker \& Humblot.

Feldstein, M.S. (2008), "Did wages reflect growth in productivity?", NBER Working Paper, $\mathrm{N}^{\circ}$ 13953, Cambridge, Massachusetts, National Bureau of Economic Research.

Fichtenbaum, R. (2009), "The impact of unions on labor's share of income: a time-series analysis", Review of Political Economy, vol. 21, $\mathrm{N}^{\circ} 4$, Taylor \& Francis.

FMI (Fondo Monetario Internacional) (2007), Informe anual 2007, Washington, D.C

Goldfarb, R.S. y T.C. Leonard (2005), "Inequality of what among whom?: Rival conceptions of distribution in the 20th century", Research in the History of Economic Thought and Methodology, vol. 23, parte 2, Emerald.

Gollin, D. (2002), "Getting income shares right", Journal of Political Economy, vol. 110, $\mathrm{N}^{\circ}$ 2, Chicago, University of Chicago Press.

Graña, J.M. (2007), Distribución funcional del ingreso en la Argentina: 1935-2005, Buenos Aires, Universidad de Buenos Aires.

Harrison, A. (2002), "The World Bank: structure and policies: Christopher L. Gilbert and David Vines (eds.), Cambridge University Press", Journal of International Economics, vol. 57, $\mathrm{N}^{\circ}$ 1, Amsterdam, Elsevier

Hernández Laos, E. (1998), "Políticas de estabilización y ajuste y distribución funcional del ingreso en México", Revista Comercio Exterior, México, D.F., Banco Nacional de Comercio Exterior.

Hogrefe, J. y M. Kappler (2012), "The labour share of income: heterogeneous causes for parallel movements?", The Journal of Economic Inequality, vol. 11, $\mathrm{N}^{\circ} 3$, Springer.

Jayadev, A. (2007), "Capital account openness and the labour share of income", Cambridge Journal of Economics, vol. 31, Nㅜ 3 , Oxford University Press.

Kaldor, N. (1961), Capital Accumulation and Economic Growth, MacMillan.

Krueger, A. (1999), "Measuring labor's share", NBER Working Paper, No. 7006, Cambridge, Massachusetts, National Bureau of Economic Research.

Lewis, W.A. (1954), "Economic development with unlimited supplies of labour", The Manchester School, vol. 22, N 2, Wiley.

Lindenboim, J. (2008), "Distribución funcional del ingreso, un tema olvidado que reclama atención", Problemas del Desarrollo. Revista Latinoamericana de Economía, vol. 39, $\mathrm{N}^{\circ} 153$, México, D.F.

Lindenboim, J., D. Kennedy y J.M. Graña (2010), "El debate sobre la distribución funcional del ingreso", Desarrollo Económico, vol. 49, ํㅜ 196, Buenos Aires, Instituto de Desarrollo Económico y Social (IDES).

Ministerio de Planificación y Cooperación (2000), Estudio sobre la distribución del ingreso: Estructura funcional en 1987-96 y proyecciones, Santiago de Chile.

OIT (Organización Internacional del Trabajo) (2013), Informe mundial sobre salarios 2012/2013. Los salarios y el crecimiento equitativo, Ginebra.

(2011), Informe mundial sobre salarios 2010/2011. Políticas salariales en tiempos de crisis, Santiago de Chile.

(2008), World of Work Report 2008. Income Inequalities in the Age of Financial Globalization, Ginebra.

Piketty, T. (2003), "Income inequality in France, 1901-1998", Journal of Political Economy, vol. 111, $\mathrm{N}^{\circ}$ 5, Chicago, University of Chicago Press.

PNUD (Programa de las Naciones Unidas para el Desarrollo) (2010), Informe sobre desarrollo humano 2010. La verdadera riqueza de las naciones: Caminos al desarrollo humano, Nueva York.

Ricardo, David (1973), Principios de economía política y tributación, Madrid, Editorial Ayuso. 
Rodríguez, O. (2006), El estructuralismo latinoamericano, Santiago de Chile, Comisión Económica para América Latina y el Caribe (CEPAL).

Rodríguez, F.R. y D. Ortega (2006), "Are capital shares higher in poor countries? Evidence from industrial surveys", Wesleyan Economics Working Papers, $\mathrm{N}^{\circ}$ 2006-023, Middletown, Wesleyan University.

Serrano, F. y C. Medeiros (2001), Economic Development and the Revival of the Classical Surplus Approach, Ciudad del Cabo.

Serres, A., S. Scarpetta y C. Maisonneuve (2001), "Falling wage shares in Europe and the United States: how important is aggregation bias?", Empirica, vol. 28, $\mathrm{N}^{\circ}$ 4, Springer.

Solow, R.M. (1958), "A skeptical note on the constancy of relative shares", The American Economic Review, vol. 98, $\mathrm{N}^{\circ} 4$, Nashville, Tennessee, American Economic Association.
Stockhammer, E. (2013), "Why have wage shares fallen? A panel analysis of the determinants of functional income distribution", Conditions of Work and Employment Series, $\mathrm{N}^{\circ} 35$, Ginebra, Organización Internacional del Trabajo (OIT).

Taylor, L. (1991), Income Distribution, Inflation, and Growth: Lectures on Structuralist Macroeconomic Theory, Cambridge, Massachusetts, The MIT Press.

Yamada, G., J. Castro y J. Bacigalupo (2012), “Desigualdad monetaria en un contexto de rápido crecimiento económico. El caso reciente de Perú", Documento de Discusión, № 12/01, Centro de Investigación de la Universidad del Pacífico.

Young, A. (1995), "The tyranny of numbers: confronting the statistical realities of the East Asian growth experience", The Quarterly Journal of Economics, vol. 110, $\mathrm{N}^{\circ} 3$, Oxford University Press. 